\title{
The polite pop-up: An experimental study of pop-up design characteristics and user experience
}

\author{
Sara Willermark \\ University West, School \\ of Buisness, Economics \\ \& IT, Sweden \\ sara.willermark@hv.se
}

\author{
Anna Sigridur Islind \\ Reykjavik University \\ School of Computer \\ Science, Iceland \\ islind@ru.is
}

\author{
Therese Appelgren \\ University West, School \\ of Buisness, Economics \\ \& IT \\ thereseappelgren95@,hot \\ $\underline{\text { mail.com }}$
}

\author{
Mia Eklund Taavo \\ University West, School \\ of Buisness, Economics \\ \& IT \\ $\underline{\text { miaeklundtaavo@hotmai }}$
}

\begin{abstract}
Pop-up boxes have been widely used to catch users' attention and highlight specific information. Yet, according to previous research, there is a high degree of perceived irritation and dissatisfaction related to pop-ups. In this study, we explore the user experience of what is referred to as "polite pop-up," i.e., a modal popup, created based on click events. The intention was to eliminate negative perceptions that pop-ups usually generate. The research method involves a constructed user test of a prototype of a website where polite popups were placed in the interface. Thirteen users participated, where most of the users noticed the polite pop-up and voluntarily chose to access the information within the pop-up. The contribution includes increased insight into the relation between polite pop-up and user satisfaction, as well as design implications for usercentered design.
\end{abstract}

\section{Introduction}

Pop-ups have been widely used as a way to catch users' attention and highlight specific information [1]. A pop-up appears on the screen as an information box and the exposure often occurs unexpectedly and independently of context [2]. Thus, pop-ups entail forced exposure, where the user is forced to interact with unwanted information before the user can return to his/her primary activity [3]. This results in cognitive costs in the form of interruption and lack of perceived autonomy. From a user perspective, pop-ups have traditionally been associated with flashing, colorful and untrustworthy squares that involuntarily appear on the screen. This type of third-party pop-up, i.e., from an external sender, was a common occurrence in the late 1990s, but decreased as browsers came to offer the alternative of filtering them out. The pop-up boxes have now returned in another form and constitute a frequently occurring phenomenon in online marketing, where the website itself is the sender of the material [4]. The original flashing and colorful pop-up boxes from third parties have diminished, although pop-up boxes are still a common feature. Even though pop-ups have been considered a powerful way to catch the user's attention [5-7], their power has often been considered so disturbing that they have been compared to distracting colleagues who insist on making conversation and therefore interrupt an ongoing task [2, 8]. Pop-ups thereby constitute a very effective "attention grabber" causing attention and reaction [5]. Due to these strong reactions, the use of pop-ups often results in a negative user experience $[5,9]$ and can cause users to leave the web page [2]. Users generally experience a high degree of irritation and dissatisfaction when exposed to pop-up boxes regardless of the design, such as the size or shape of the pop-up box or when they appear on the screen [2]. Thus, there is a conflict of interest between the designers' desire to influence and control the users' attention on the one hand, and the user experience of pop-ups on the other hand [5], a conflict which we explore in this paper.

To sum up, previous research shows that pop-ups constitute an effective way of attracting attention as well as controlling the user's behavior. However, they have been known to cause negative user experience. The purpose of the study is to explore the user experience of so-called "polite pop-ups" that are implemented into the interface of a website where the intrusive and surprising factors are eliminated. A traditional pop-up is a box or a window that suddenly pops up and requires the user to select an option with mouse or screen interaction to be able to proceed. The polite pop-up does however differ, as it is a part of the website itself. The user is not forced 
into e.g. mouse-interaction to be able to proceed, as the polite pop-up can be scrolled over without interaction. It deviates from other parts of a website as its content can be accessed by clicking and it does differ visually from other elements of the website without causing cognitive overload. Thus, a polite pop-up is a modal pop-up, created based on click events. The research question is as follows: "What is the user experience of a polite pop-up implemented at an e-commerce website?"

\section{Theory}

Different types of pop-ups have been developed and applied in various ways. The different types are exposed to the user in alternating ways, such as "timed pop-ups," "scroll pop-ups," "entry pop-ups," "exit pop-ups” and "click pop-up" [7]. "Timed pop-ups" are activated after a certain time on the website, for example, 60 seconds, while "scroll pop-ups" are activated when the user has scrolled down the page to a certain length. "Entry popups" are activated as soon as the landing page (i.e., a page the user arrives at) is fully loaded, and thus is exposed directly to the user, and "exit pop-ups" are activated when the user is leaving the page. "Click popups" are activated when clicking on a specific link, image or word and constitute the only pop-up where the interaction is not imposed on the user. In addition to these five pop-up types, we have identified a type called "time-limited pop-up" that arises as a confirmation of an event and disappears automatically after a short time span without any user interaction. However, "click popups" constitute the focus of this study as it is the only pop-up type where the interaction is not imposed on the user. Tasse, Ankolekar and Hailpern [5] have suggested that pop-ups could be placed in the interface within the visual field without the requirement for active action to avoid negative experiences related to pop-ups. However, eliminating forced interaction and the dynamic element will most likely be at the expense of bringing attention to the pop-up boxes. Nevertheless, they argue that there are reasons to believe that it would constitute a more appreciated and accepted way to influence users [5]. That assumption, and the lack of research regarding that assumption, is the basis for our study.

\section{Research Approach}

The overall research approach involves both qualitative and quantitative data to get a rich understanding of user experience during the exposure to polite pop-ups. We have chosen to limit the investigation to "click pop-ups" since previous research shows consistent results of traditional pop-ups as negative [2, 1, 9]. Click pop-ups, on the other hand, are relatively unexplored, and in this case, we explore polite pop-ups (modal popup) that are implemented into the interface of a website, based on click events. The research method involves 1) a constructed user test; 2), observation of user test; and 3) an assessment questionnaire. These data collection activities were mixed to gather different aspects of the user experience. The data collection activities were conducted in the given order and during the same occasion. The participants were initially informed that they would test and evaluate a website prototype. They were asked to navigate the website and answer a questionnaire. However, they were not informed of the role of the pop-up as it would guide the participants' focus and inhibit the authenticity of the situation.

\subsection{Participants}

The study included 13 participants based on availability and willingness to participate. All participants attended a university in Sweden, however, age (from 18 to 30 years old) and study orientation varied. Participants were recruited through an open recruitment process that involved advertising for participants on digital screens at the university as well as advertising on social media platforms connected to the university. The participants thereby willingly signed up for participation and were not targeted or approached specifically.

\subsection{User Test}

An e-commerce website prototype was developed for the specific purpose of the study. The purpose of the prototype was to promote the experience of an authentic website that users wanted to engage in (see Figure 1), by attempting an easy-to-navigate and visually attractive website [10]. Hoping to generate interaction with polite pop-ups, a prototype containing three main views was created including 1 ) a start page; 2) category pages; and 3 ) product pages. The prototype was of an e-commerce site that sold clothes. During the prototype navigation, the user first came to the start page. Clicking on a product on the category page led to the product page, which showed specific products. The fact that the prototype was an e-commerce site was not the focus of this study, it was merely the means of testing the click pop-ups in a setting that is familiar to most users. The color and shape of the click pop-ups differed visually from the surrounding environment (see Figure 1). This was to stimulate the visual perception ability that triggers memory, making the information easier to recall $[1,11]$.

The users could be exposed to three polite pop-ups, scattered in the interface of the different views. The polite pop-ups consisted of a modal dialog box, where the modal popup appears to make the rest of the web page inaccessible until the popup is closed, and were created based on click events. The pop-ups were 
designed with the ambition to be in line with the overall web design in order to not be perceived as intrusive. These were click pop-up bait including: a) best-selling products on the two category pages (see Figure 2); b) discount code on the start page (see Figure 3); and c) a contest with a possibility of a prize on the category page as well as on the start page (see Figure 4). The click popups could be dismissed by clicking on an X.

The user test was designed so that all participants were exposed to and asked to navigate on the prototype according to identical instructions. The participants were requested to navigate as consumers on an ecommerce website. By giving the participants a clear directive with specific tasks to perform, the participants navigated within the website and were exposed to polite pop-ups three times. The user tests were conducted in a private room to restore as much of a natural environment as possible. We camouflaged the user test so that participants were not aware of its primary purpose and thus did not actively reflect on the perception of polite pop-up [13]. We communicated the real purpose as soon as the study was completed.

The prototype was developed in the Adobe XD CC software program and the user tests were conducted on a laptop. The user tests were carried out in May 2018.

The observation was documented by video recording of the participants' screen. To explore the users' interaction and behavior related to the polite popups screen recording was applied.

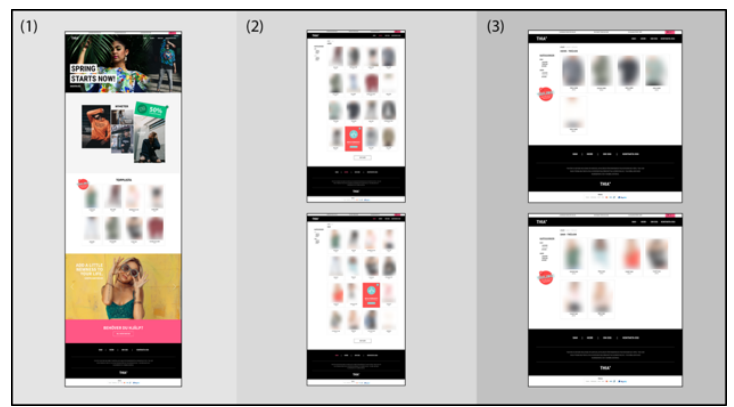

Figure 1. An overview of the prototype design containing the polite pop-ups.

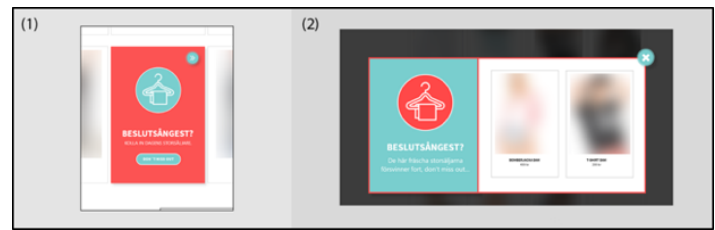

Figure 2. Illustration of pop-up 1, "Best seller", before clicking (no. 1) and the result of click (no. 2).

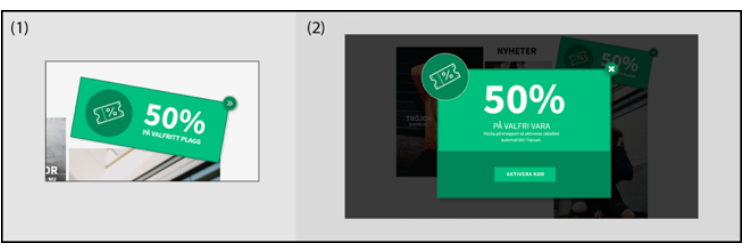

Figure 3. Illustration of pop-up 2, "Discount", before clicking (no. 1) and the result of click (no. 2).

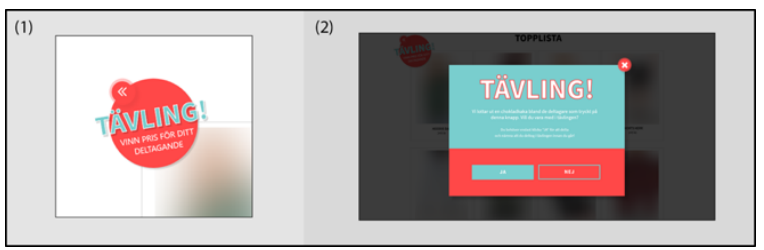

Figure 4. Illustration of pop-up 3, "Contest", before clicking (no. 1) and the result of click (no. 2).

\subsection{Assessment Questionnaire}

In conjunction with the user test and observations the participants were asked to fill out an assessment questionnaire. Prior to the questionnaire, the participants were assigned a pseudonym, to link the questionnaires and screen recordings to a specific person without their real name showing in the data. Thus, none of the names presented in the results are the real names of the participants. The questionnaire included 10 questions regarding the prototype in general and the polite pop-ups in particular, to explore the user experience of the prototype. The questions were answered by multiple choice. If the participants did not experience that any of the alternatives were suitable, there was a supplementary response field to fill in their results.

\subsection{Analysis}

The data analysis involves both observational data and the questionnaire. The observational data was analyzed with an observation protocol based on what actions participants made (navigation pattern and clicks) as well as the frequency and duration of different actions. Further, we explored whether the participants chose to dismiss the click pop-up after activating it, or to take further actions, that is, participate in the suggested contest or use a discount code, which is referred to as "active engagement." Furthermore, the questionnaire was coded based on all the participants' responses, regarding experienced behavior in interaction with, and preferences of, the prototype. In addition, the relationship between the participants' 
responses in the questionnaire and their actions in the observation was also coded.

\section{Results}

Among the thirteen participants in the study, nine $(69 \%)$ interacted with one or more click pop-ups. The other four participants (31\%) did not interact with any polite pop-ups. A total of five participants interacted with more than one polite pop-up. The average time the participants were exposed to the clicked information was 6.2 seconds.

Regarding the interaction with the prototype in general, the largest degree of interaction took place on the product and category pages whereas the start page tracked less attention than the other specific pages.

Regarding the interaction with the polite pop-ups in particular, the three different polite pop-ups contained information on different types of bait: 1) information on best-selling products; 2) a discount code; and 3) a contest with a possibility of a prize.

\subsection{Polite Pop-up 1}

Polite pop-up 1 contained information on bestselling products (see Figure 5) that the participants encountered when navigating the category pages for women and men.

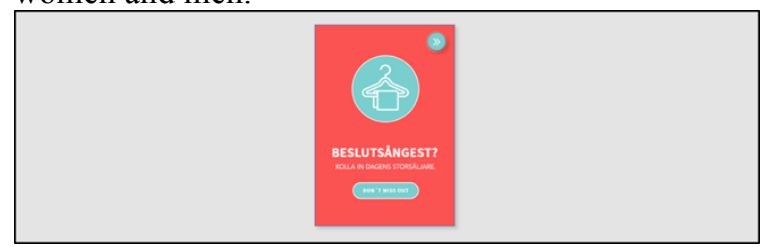

Figure 5. Illustration of polite pop-up 1. "Best seller."

\begin{tabular}{|l|l|}
\hline Interaction with the pop-up & Total \\
\hline Number of clicks & 6 \\
\hline Average time & $5.2 \mathrm{sec}$ \\
\hline Number of repeated clicks & 1 \\
\hline Active engagement & 5 \\
\hline
\end{tabular}

\section{Table 1. Degree of interaction with polite pop- up $1(n=13)$.}

Table 1 shows the total number of participants who chose to interact with polite pop-up 1. Five participants interacted, resulting in a total of $38 \%$. Among these five, one participant clicked on the polite pop-up placed on the women's category page. The remaining four clicked on the one that was placed on the category page for men. The average time the participants were exposed to the boxes was 5.2 seconds. Regarding the two different category pages, there was a difference concerning the exposure time, where the content of the box on the men's page was considered the longest. On the category page for men, there was also a higher degree of active engagement and interest shown, as all participants clicked onto a product that appeared to be a great seller. What is considered an active engagement here is when the participants took further actions like wanting to explore the best-selling products.

\subsection{Polite Pop-up 2}

Polite pop-up 2 presented a discount code (see Figure 6) that the participants could find when navigating on the start page.

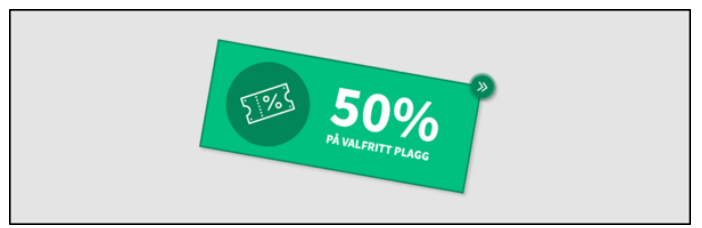

Figure 6. Illustration of polite pop-up 2. "Discount."

\begin{tabular}{|l|l|}
\hline Interaction with the pop-up & Total \\
\hline Number of clicks & 1 \\
\hline Average time & $4 \mathrm{sec}$ \\
\hline Number of repeated clicks & 0 \\
\hline Active engagement & 1 \\
\hline
\end{tabular}

Table 2. Degree of interaction with polite popup $2(n=13)$.

Table 2 presents the total number of participants who chose to interact with polite pop-up 2. Only one participant interacted, resulting in $8 \%$ active engagement. The time that the participant was exposed to the box was 4 seconds. After noticing the content, the participant chose to activate the discount code by clicking the button "ACTIVATE CODE" that was included in the box, showing signs of genuine engagement in the content.

\subsection{Polite Pop-up 3}

Polite pop-up 3 contained an offer to participate in a competition (see Figure 7). The participants could find 
this pop-up both while navigating on the start page and while navigating on the category pages.

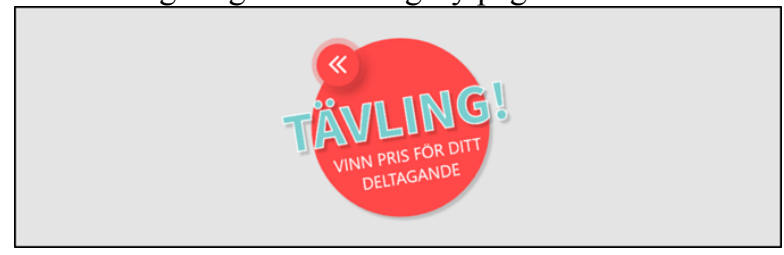

Figure 7. Illustration of polite pop-up 3 "Contest."

\begin{tabular}{|l|l|l|l|}
\hline $\begin{array}{l}\text { Interaction with } \\
\text { the pop-up }\end{array}$ & $\begin{array}{l}\text { Start } \\
\text { page }\end{array}$ & $\begin{array}{l}\text { Category } \\
\text { page }\end{array}$ & Total \\
\hline Number of clicks & 1 & 9 & 10 \\
\hline Average time & $8 \mathrm{sec}$ & $9 \mathrm{sec}$ & $8.7 \mathrm{sec}$ \\
\hline $\begin{array}{l}\text { Number of } \\
\text { repeated clicks }\end{array}$ & 0 & 2 & 2 \\
\hline $\begin{array}{l}\text { Active } \\
\text { engagement }\end{array}$ & 1 & 5 & 6 \\
\hline $\begin{array}{l}\text { Distribution of } \\
\text { active engagement }\end{array}$ & $13 \%$ & $87 \%$ & $100 \%$ \\
\hline
\end{tabular}

Table 3. Degree of interaction with polite pop-up $3(n=13)$.

Table 3 shows the total number of participants who chose to interact with click pop-up 3. Eight participants interacted, resulting in a total of $62 \%$. Among these eight, only one participant clicked on the polite pop-up that was placed on the start page, which correlates with the general results of less time spent on the start page. The remaining seven participants clicked on the polite pop-ups that were placed on the category pages. The average time the participants were exposed to the boxes was 8.7 seconds. Regarding exposure on the two different category pages (men's and women's), the exposure time was relatively equal (with no significant difference), whereas the category page click pop-up was considered slightly longer in relation to the one placed on the start page. With this polite pop-up, a relatively higher degree of active engagement and interest shown was identified, as most participants stated that they wanted to take part in the competition and clicked "YES" on the click pop-up. Interestingly, two participants clicked "NO" on the click pop-up and chose not to participate in the competition but chose to click anyway.

\subsection{User Experience of Click Pop-ups}

Most participants stated that they were met by some type of component that triggered the pop-up boxes during their navigation on the website. In the questionnaire, a majority stated that they had seen polite pop-ups 1 and 3 . Of the participants, $62 \%$ stated that the way in which the information was presented, i.e., that on their own initiative they had to click on the information, was good because they themselves were able to choose whether to view the information, as illustrated by "Clearly and well presented..." (Martin) and "Good and alluring offers but not thrown into your face" (Helena).

Regarding the question of whether they frequently took advantage of best-selling products, used discount codes, or participated in competitions on e-commerce websites if these options are available, the majority answered yes. This indicates that the participants, during observation, took note of elements that they usually notice when browsing such websites. None of the participants stated that they encountered an unexpected outcome when they clicked on a polite pop-up. Of the participants, $69 \%$ stated that they had not wanted the same information to have been presented automatically (i.e., in a regular pop-up), while $23 \%$ stated that they were not annoyed by traditional pop-ups and $8 \%$ did not know.

Regarding the overall UX of the website, $69 \%$ of the participant stated that the color and shape mainly caught their attention on the user interface components, $23 \%$ stated that it was the text content and $8 \%$ stated the placement was the main contributor. Amir explains that the polite pop-up "lights up in front of you," while Karin expressed "enticing and helpful images, which provided information and tips for [enriching the experience] of the website." Johann did not interact with any polite pop-up and explained that he assumed that the site required contact information and thus chose not to engage by clicking.

The answers in the questionnaire show that eight participants claim that they have interacted with polite pop-up 1 in the interface, which constitutes $62 \%$. Furthermore, six participants claimed that they had been met by polite pop-up 1 and the information included in this via click. Despite this, the video material shows that only five participants interacted with it. Livia claimed that the word "indecision" (having a hard time deciding) that was included in polite pop-up 1 triggered an interest in the content, which resulted in interaction: "like you would get help finding something that fits you!"

The answers from the questionnaire show that two participants claim that they noticed polite pop-up 2 in the interface, which is merely $15 \%$ of all. Helena reported seeing polite pop-up 2 and the information in it via a click, which is consistent with what could be 
distinguished in the video material. This participant explains his actions according to the following quote: "Discount is always something that helps to convince me when I shop. I often look for numbers and other offers and it can be crucial for whether I buy or not, and from which website."

The answers from the questionnaire show that eleven participants claim that they saw polite pop-up 3 in the interface, which is $85 \%$ of all. Furthermore, seven participants claimed that they were met by polite popup 3 and the information in this via click. Despite this, the video material shows that eight participants interacted with the polite pop-up, which shows that one participant who has been exposed to the information did not state that it happened. Livia explains the action of clicking the polite pop-up as follows: "mostly to see what the prices were, I was not necessarily interested in participating" and Martin said, "A competition is something you always check out."

\section{Discussion}

Design constitute an established interest within information systems $[12,13]$. However, little attention has been drawn towards user experience of alternative pop-up design. In previous research regarding pop-ups there is a high degree of perceived irritation, dissatisfaction and low commitment to pop-ups [2]. Conversely, the results of this study point to a high degree of commitment (69\%) for the polite pop-ups. The results indicate a positive attitude towards our polite pop-ups, which differ from the pop-up boxes that are included in previous research. The active engagement shown in this study contradicts previous studies that state that pop-ups are only perceived as interruptions that are ignored by users [2]. In the questionnaire, we could see a hint of a positive attitude towards information that is presented automatically, i.e., traditional pop-ups. The fact that the term "pop-ups" was omitted from the question and the notion of automatically presented information was used instead may have affected the participants' positive response in line with the arguments made by Tasse, Ankolekar and Hailpern [5] where they discuss that the concept often has negative connotations.

In contrast to Bahr and Ford's study [2], where the participants associated pop-ups with information they did not want to see and began rejecting them, our study did not reveal such results. Five of the nine participants who interacted with any of the polite pop-ups returned to another polite pop-up on the site, or visited the same one again. That particular behavior pattern, and active engagement, can be interpreted as either i) that the participants needed clarification, which led to a return visit to the same box, or ii) that the information they previously encountered in one polite pop-up was interesting and resulted in interacting with several. The notion that the polite pop-up was not rejected by the participants is based on the fact that they stayed at the polite pop-up long enough to process the information and evaluate the content. The mean of the time span was 6.2 seconds in our study, which can be compared to Bahr and Ford's [2] claim that it takes about 1.3-1.5 seconds for the user to take in the contents of a pop-up. A reasonable assumption is thereby that the participants certainly took in the content as they were exposed to the polite pop-ups for a sufficiently long time, which indicates a great probability that they considered the content worth spending time on.

Polite pop-up 3 attracted the highest degree of active engagement. Factors that may have influenced the decision to click on the polite pop-up may be influenced by the fact that its form was distinct in comparison to the surrounding material and that it was available on three places on the website while the two other polite pop-ups were placed on two pages (for men and women). However, only one participant clicked on polite pop-up 1 on the start page. The explanation for the higher degree of engagement can also be that the competition triggered the participant's personal interest, as the purpose of this pop-up was the only polite pop-up that presented a physical prize, outside of the website's content. The two other polite pop-ups (best-selling products and discount codes) therefore most likely did not generate as high a sense of profitability. Pop-ups and their contents need to be coherent with the user's current task to avoid negative reactions [1] which is a likely cause of the high click-through rate.

Polite pop-up 2 tracked the lowest degree of active engagement. This polite pop-up was distinguished in color from the other two click pop-ups and the color of this click pop-up might have been harder to see due to the surrounding colors (and it thereby blended in more). Regarding the location, a decision was made to apply the click pop-up high up on the start page, in line with Roth et al. [14] who argue that an element is more likely to be detected if it is placed high up. However, in this study that particular location was not the case. This might correlate with the general lack of interest in our start page as a whole, which is based on the observations of the navigation on the website prototype. Roth et al. [14] further explain that elements placed on the left side are more likely to be noticed in comparison with the right side, and polite pop-up 2 was placed to the right whereas the other polite pop-ups were placed either in the middle or to the left.

As the participants, on their own initiative, could access the information, the outcome was as expected and in line with previous research on pop-ups that demonstrate how more invasive pop-ups can be perceived as intrusive [2]. Choosing whether to access 
the information can be seen as a more modern, polite way of acting on the Internet. With regards to the interaction design, the users in our study pointed out that color and shape of the polite pop-ups constituted the main reason for them noticing the polite pop-ups, which confirms that appearance of the elements in the polite pop-up and the user experience and interaction design of the pop-ups should be in contrast to the remaining content of the website in order for them to be discovered. We conclude that our study indicates that polite pop-ups work better than traditional invasive popups both when it comes to active engagement as well as in relation to ability to recall the information that the users are exposed to.

\subsection{Design Implications}

The design implications that can be drawn from the results of this study indicate that when designing popups, the recommendation is a less invasive and more friendly design because these pop-ups are more likely to trigger interaction than traditional pop-ups. It can be used without cognitive costs in the form of interruption and lack of perceived autonomy. The results also indicate that the users interact with polite pop-ups if the content is of value for them. We are entering a new era of pop-ups where the polite pop-ups are not perceived as irritating to users, and they trigger an active engagement when the content of the pop-up is perceived as valuable for the users. We suggest that the polite popup should be in line with the overall web design to not be perceived as intrusive. It is at the expense of the fact that not all users will pay attention to the polite pop-up. Yet, the results from this study show that most of the users noticed the polite pop-up and willingly chose to access the information within the pop-up, i.e., proved susceptible.

\subsection{Limitations}

The study primarily has three limitations that should be noted, and that also can serve as areas for future research. First, the user test constitutes a constrained situation and thus direct application of study findings into real-life situations should be carried out with caution. However, measures were taken to create as authentic a situation as possible; the experimental setting constituted an environment that participants are familiar with and the activity carried out in the user test was realistic. This was to create as representative a sequence of events as possible in an otherwise constructed situation [14]. Second, the data is limited in terms of sample size and lack of a separate control group, and thus further studies are needed to determine results to be able to draw generalizable assumptions. Third, there was a lack of dynamic elements in our click pop-ups, which research claims will result in reduced attention to the pop-ups [5]. We are aware that this factor may have affected the results of our study as well as imposed severe restrictions on what conclusions can be drawn.

\section{Conclusion}

We have explored the user experience of what is referred to as "polite pop-ups." Our results show that polite pop-ups can be used as a substitute for traditional pop-ups, and thus reduce negative perceptions from the user. The results of our study show that users willingly interacted with polite pop-ups for a relatively long time, and our study therefore concludes that polite pop-ups constituted a fruitful way to both catch users' attention and avoid user dissatisfaction. 


\section{References}

[1] Bittner, J. V., and R. J. Zondervan, "Motivating and achievement-eliciting pop-ups in online environments: A user experience perspective", Computers in Human Behavior, Vol. 50, 449-455, 2015.

[2] Bahr, G. S., and R. A. Ford, "How and why pop-ups don't work: Pop-up prompted eye movements, user affect and decision making", Computers in Human Behavior, 27(2), 776-783, 2011.

[3] Edwards, S. M., H. Li, and J-H. Lee, "Forced exposure and psychological reactance: Antecedents and consequences of the perceived intrusiveness of pop-up ads", Journal of Advertising, 31(3), 83-95, 2002.

[4] Ratner, B. "Should marketers use popup forms? A comprehensive analysis." Hubspot [Blog] Published on 11 October 2018. Available on:

https://blog.hubspot.com/marketing/pop-up-forms-analysis. [Accessed on: 2019-01-19].

[5] Tasse, D., A. Ankolekar, and J. Hailpern, "Getting Users' Attention in Web Apps in Likable, Minimally Annoying Ways", in Proceedings of the 2016 SIGCHI Conference on Human Factors in Computing Systems, 3324-3334, ACM, 2016.

[6] Dabbish, L., G. Mark, and V. M. González, "Why do I keep interrupting myself?: environment, habit and selfinterruption", in Proceedings of the 2011 SIGCHI Conference on Human Factors in Computing Systems. ACM, 2011.

[7] Warnaby, G., and C. Shi, Pop-up Retailing: Managerial and Strategic Perspectives. Heidelberg: Springer, 2018.

[8] Leroy, S., "Why is it so hard to do my work? The challenge of attention residue when switching between work tasks", Organizational Behavior and Human Decision Processes, 109, (2), 168-181, 2009.

[9] Chatterjee, P., “Are Unclicked Ads Wasted? Enduring Effects of Banner and Pop-Up Ad Exposures on Brand Memory and Attitudes", Journal of Electronic Commerce Research 9.1, 2008.

[10] Hasan, B., "Perceived irritation in online shopping: The impact of website design characteristics", Computers in Human Behavior, 54, 224-230, 2016.

[11] Diao, F, and S. Sundar, "Orienting response and memory for web advertisements: Exploring effects of popup window and animation", Communication Research, $31.5,2004$.

[12] Willermark, S., Digital Didaktisk Design: Att utveckla undervisning $i$ och för en digitaliserad skola (Doctoral dissertation, University West), 2018.
[13] Islind, A. S., Platformization: co-designing digital platforms in practice (Doctoral dissertation, University West), 2018

[14] Roth, S. P., A. N. Tuch, E. D. Mekler, J. A. BargasAvila, \& K. Opwis, "Location matters, especially for nonsalient features-An eye-tracking study on the effects of web object placement on different types of websites", International Journal of Human-Computer Studies, 71(3), 228-235, 2013.

[15] Bryman, A., Social Research Methods. Oxford University Press, 2016. 